\title{
Consultores e Revisores
}

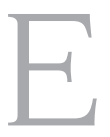

m nome da comunidade de reumatologistas brasileiros, os editores da Revista Brasileira de

Reumatologia (RBR) agradecem aos consultores aqui relacionados e aos membros do Conselho Editorial a importante contribuição realizada em 2007.

A revisão dos manuscritos, feita de maneira minuciosa e por profissionais especializados, é essencial para conferir integridade e confiabilidade à publicação científica. Não custa lembrar que esse é um trabalho voluntário e, pela própria natureza, anônimo.

Merecem um comentário e agradecimento especial os colegas que, mesmo não fazendo parte do Corpo Editorial, têm contribuído de forma regular e criteriosa com a RBR, empregando parte do seu tempo e conhecimento no processo de revisão por pares.

Por intermédio da dedicação e do comprometimento desses profissionais é que a RBR alcança hoje qualidade e regularidade de publicação.

Estamos em um momento de reflexão sobre os procedimentos a serem adotados para melhorarmos ainda mais a qualidade da nossa revista. A revisão do Corpo Editorial, que segue uma periodicidade, deverá buscar adequar-nos para conseguir nosso maior objetivo nesse momento, que é a necessária indexação ao Medline.

A esses profissionais deixamos os nossos sinceros agradecimentos por seu valioso trabalho.

Francisco Airton Castro da Rocha e

Ricardo Machado Xavier

Editores Científicos
Alexandre Wagner S. de Souza

Angela Luzia Branco P. Duarte

Blanca Elena Rios Gomes Bica

Boris Afonso Cruz

Charles Heldan de Moura Castro

Eduardo Antonio Donadi

Eduardo dos Santos Paiva

Elda Matilde Hirose Pastor

Fernando de Souza Cavalcanti

Francisco Rafael Laurindo

Ieda Maria Magalhães Laurindo

Ivanio Alves Pereira

Izaias Pereira da Costa

Jose Carlos Mansur Szajubok

José Tupinambá

Jozelio Freire de Carvalho

Manoel Barros Bértolo

Marcelo de Medeiros Pinheiro

Maria José Pereira Vilar

Maria Teresa S. L. R. A. Terreri

Mauro Waldemar Keisermann

Paulo Louzada Jr.

Percival D. Sampaio-Barros

Roberto Ezequiel Heymann

Rosa Maria Rodrigues Pereira

Sergio Candido Kowalski

Tatiana Freitas Tourinho

Valderilio Feijo Azevedo

Valéria Valim

Virginia Fernandes Moça Trevisani 